PROCEEDINGS OF THE

AMERICAN MATHEMATICAL SOCIETY

Volume 134, Number 6, Pages 1849-1856

S 0002-9939(05)08185-2

Article electronically published on December 15, 2005

\title{
ON CHARACTER GROUPS ARISING FROM DIMENSION GROUPS
}

\author{
RYAN J. ZERR
}

(Communicated by Joseph A. Ball)

\begin{abstract}
Character groups associated with certain dimension groups are considered. It is shown how these character groups can be used to construct an $\mathrm{AF}$ groupoid whose dimension group is isomorphic to the original dimension group.
\end{abstract}

\section{INTRODUCTION}

A class of algebras of great importance in operator theory are the approximately finite-dimensional (AF) $\mathrm{C}^{*}$-algebras. These algebras are inductive limits of finitedimensional $\mathrm{C}^{*}$-algebras $\mathfrak{A}_{n}$, and in this sense might be considered the next simplest types of $\mathrm{C}^{*}$-algebras after the finite-dimensional algebras themselves. The $\mathrm{AF}$ algebras have been widely studied, and it was Elliott 2] who established a bijective correspondence between unital AF algebras and certain ordered groups with order units, called dimension groups. These dimension groups are defined in terms of the $K$-theory of the subalgebras $\mathfrak{A}_{n}$, and are inductive limits of groups of the form $\mathbb{Z}^{k_{n}}$. Dimension groups provide one vantage point in the study of AF algebras.

An alternative vantage point is provided within the theory of groupoids. Informally, a groupoid is a set with a partially defined multiplication for which the usual properties of a group hold whenever they make sense. In the context of AF algebras, these groupoids are topological equivalence relations on which the partially defined multiplication is described by the transitivity of the relation. In [6] it is shown how to use an AF algebra to build such an equivalence relation groupoid. For example, in the case of the algebra $M_{n}$ of $n \times n$ complex matrices, this groupoid amounts to working with the basic building blocks, the matrix units $e_{i j}$, and multiplying them in the usual way, ignoring the cases where the product is 0 . A groupoid arising in this way is an example of an AF groupoid. There is an algebra of continuous, complex-valued functions defined on such a groupoid, and this algebra (see [4, 7]) is isomorphic to the original AF algebra. So, there is also a bijective correspondence between AF algebras and $\mathrm{AF}$ groupoids, and consequently between $\mathrm{AF}$ groupoids and dimension groups via the intervening AF algebra.

It is desirable to have a more direct relation between AF groupoids and their dimension groups, and progress toward making such a connection is provided in [5].

Received by the editors January 10, 2005 and, in revised form, January 28, 2005.

2000 Mathematics Subject Classification. Primary 22A22, 46L80, 47L40.

Key words and phrases. AF $\mathrm{C}^{*}$-algebras, dimension groups, character groups.

(C)2005 American Mathematical Society Reverts to public domain 28 years from publication 
There the means for using an arbitrary AF groupoid's structure to build the associated dimension group are described. Preliminary work is also mentioned regarding the inverse process - building the groupoid associated to a given dimension group. This paper is meant to expand on some of the work done in [5] by further developing a Pontryagin-like duality for certain dimension groups.

Recall, given a group $G$, a character of $G$ is a homomorphism from $G$ into the group $\mathbb{T}$, the unit circle in the complex plane. If the group $G$ is also a topological group, then the character group of $G$ is the group of all continuous characters of $G$. Given a certain type of dimension group, this paper will describe an associated character group whose structure can easily be used to build a groupoid. In fact, given a dimension group $G$ with order unit $u$, this will be the character group of the quotient group $G /\langle u\rangle$. This character group will then serve as the unit space of a groupoid (for us, the diagonal of an equivalence relation), with the elements of the group acting as partial homeomorphisms on the unit space. These partial homeomorphisms will form an inverse semigroup whose graphs can then be used to build the desired groupoid. In this way the elements of the character group will provide an analogue to the matrix units mentioned above. We will then show that the groupoid so constructed has an AF algebra whose dimension group is isomorphic to the one with which we started, thus completing the circle of ideas.

\section{Preliminaries}

Consider the dimension group $\left(G, G^{+}, u\right)$, and let $P=G /\langle u\rangle$, where $\langle u\rangle$ is the subgroup of $G$ generated by the order unit $u$. In this paper we will be considering the situation when the character group $\widehat{P}$ of $P$ is the unit space of an AF groupoid. For this to occur, it must be that $\widehat{P}$ is compact, which is equivalent to $P$ being discrete. Furthermore, it will also be necessary that $\widehat{P}$ have a basis consisting of sets which are simultaneously open and closed (clopen). That is, it will be necessary that $\widehat{P}$ be 0 -dimensional. But this is equivalent to $P$ being a torsion group. Therefore, the necessary condition that $\widehat{P}$ be a 0 -dimensional compact Hausdorff space is equivalent to $P$ being a discrete torsion group. We summarize these observations in the following lemma.

Lemma 2.1. Let $\left(G, G^{+}, u\right)$ be a dimension group. Then, $P=G /\langle u\rangle$ is a discrete torsion group if and only if $\widehat{P}$ is a 0 -dimensional compact Hausdorff space.

As a consequence of these observations, in this paper we will work with those dimension groups such that $P$ is a discrete torsion group.

A partially ordered group $\left(A, A^{+}\right)$is said to be unperforated if whenever $a \in$ $A, k \in \mathbb{Z}^{+}$, and $k a \in A^{+}$, then $a \in A^{+}$. The fact that dimension groups are unperforated [1] allows us to conclude the following, whose proof we leave to the reader.

Lemma 2.2. Let $\left(G, G^{+}, u\right)$ be a dimension group such that $P$ is a torsion group. Then, $G$ is linearly ordered.

Now, taking $g \in G^{+}$, assume that $g \geq u$. By definition of an order unit, for $N$ sufficiently large, $N u>g$. If we let $n+1$ be the smallest such positive integer, then due to the fact that $G$ is linearly ordered, there is a largest positive integer $n$ such that $n u \leq g$. So, $0 \leq g-n u<u$, and in $P,(g-n u)+\langle u\rangle=g+\langle u\rangle$. A similar argument shows that for $g<0$, there exists a greatest positive integer $n$ such that 
$g<-n u$. Then, $0 \leq g+(n+1) u<u$, and in $P, g+(n+1) u+\langle u\rangle=g+\langle u\rangle$. Consequently, every element of $P$ can be represented uniquely as $g+\langle u\rangle$, where $0 \leq g<u$ in $G$. We are now equipped to prove the next lemma.

Lemma 2.3. Assume $B$ is a clopen subgroup of $\widehat{P}$. Then, $\widehat{P} / B$ is a finite cyclic group.

Proof. We define the subgroup $A(P, B) \subset P$ by

$$
A(P, B)=\{x \in P: \chi(x)=1, \text { for all } \chi \in B\} .
$$

By [3, Thm 23.25] and the Pontryagin duality theorem, it follows that the character group of $\widehat{P} / B$ is isomorphic to $A(P, B)$. Then, since $B$ is a clopen subgroup of $\widehat{P}$, $A(P, B)$ is compact in $P$. As $P$ has the discrete topology, we are led to conclude that $A(P, B)$ is a finite subgroup of $P$. The result then follows from the fact that every element of $P$ can be represented uniquely as $g+\langle u\rangle$, where $0 \leq g<u$ in $G$.

\section{Character partial homeomorphisms}

We will now be interested in using the character group $\widehat{P}$ to construct a certain inverse semigroup. The means for doing so when $P$ is infinite will be significantly more involved than for the finite $P$ case. As such, we begin by briefly mentioning the finite case.

Again utilizing the fact that every element of $P$ can be represented uniquely as $g+\langle u\rangle$ where $0 \leq g<u$ in $G$, we conclude that there exists an $m \in \mathbb{Z}^{+}$ such that $P \cong \mathbb{Z}_{m}$, and consequently, $\widehat{P} \cong \mathbb{Z}_{m}$. By a partial homeomorphism $\sigma$ on a topological space $X$ we mean that $\operatorname{Dom}(\sigma)$ and $\operatorname{Ran}(\sigma)$ are open subsets of $X$, and $\sigma$ is a homeomorphism from $\operatorname{Dom}(\sigma)$ to $\operatorname{Ran}(\sigma)$. From $\widehat{P}$ we build an inverse semigroup of partial homeomorphisms simply by defining, for each $\chi \in \widehat{P}$, the mapping which takes $\chi_{a}$ to $\chi \chi_{a}$, where, since $\widehat{P}$ has the discrete topology, $\chi_{a}, \chi \chi_{a} \in \widehat{P}$ are clopen sets in $\widehat{P}$. The collection $\left\{\chi: \chi_{a} \rightarrow \chi_{a} \mid \chi, \chi_{a} \in \widehat{P}\right\}$ of all such partial homeomorphisms forms an inverse semigroup of partial mappings under composition as in [4]. The associated groupoid [6], being the union of the graphs of these mappings, will be the set

$$
\mathcal{R}=\left\{\left(\chi_{a}, \chi \chi_{a}\right): \chi, \chi_{a} \in \widehat{P}\right\}=\left\{\left(\chi_{a}, \chi_{b}\right): \chi_{a}, \chi_{b} \in \widehat{P}\right\}=\widehat{P} \times \widehat{P} .
$$

It is clear in this case that the associated $\mathrm{C}^{*}$-algebra, $C^{*}(\mathcal{R})$, is simply $M_{m}$, the $m \times m$ matrices. The dimension group of $C^{*}(\mathcal{R})$ in this case is then isomorphic to $\left(\mathbb{Z}, \mathbb{Z}^{+}, m\right)$. Since $P \cong \mathbb{Z}_{m}$ and $G$ is linearly ordered, it must be that $G$ is an infinite cyclic group with generator equal to the element $1 \in \mathbb{Z}_{m} \cong P$. Hence, $G \cong \mathbb{Z}$, and in fact, it is then clear that $\left(G, G^{+}, u\right) \cong\left(\mathbb{Z}, \mathbb{Z}^{+}, m\right)$.

So, in summary, when $P$ is finite, the character group $\widehat{P}$ gives rise, in a natural way, to an inverse semigroup of partial homeomorphisms on $\widehat{P}$, and by extension, to a groupoid with unit space $\widehat{P}$. This groupoid is then such that the associated $\mathrm{C}^{*}$-algebra is an AF algebra whose dimension group is $\left(G, G^{+}, u\right)$. The rest of our efforts will be devoted to generalizing this result to the case when $P$ is infinite.

Assuming $P$ is infinite, consider the sequence $\left\{A_{n}\right\}_{n=1}^{\infty}$ of open sets in $\widehat{P}$ with, for each $n \geq 1$,

$$
A_{n}=\left\{\chi \in \widehat{P}:|\chi(x)-1|<1 / n \text { for all } x \in\left\{a_{1}, a_{2}, \ldots, a_{n}\right\}\right\},
$$


where $a_{1}, a_{2}, \ldots$ is an enumeration of $P$. It follows that $\bigcap_{n=1}^{\infty} A_{n}=\{\operatorname{id}\}$, where id $\in \widehat{P}$ is the character $\operatorname{id}(x)=1$, for all $x \in P$. The fact that $\widehat{P}$ is 0 -dimensional means we can find a nested decreasing sequence $\left\{\bar{B}_{n}\right\}_{n=1}^{\infty}$ of basic clopen sets such that $\bigcap_{n=1}^{\infty} \bar{B}_{n}=\{\mathrm{id}\}$. Note that if $P$ were finite, this sequence would eventually be constant, whereas here we may assume without a loss of generality that it is strictly decreasing.

By [3, Thm 7.5], there exists a clopen subgroup $B_{1} \subset \bar{B}_{1}$ in $\widehat{P}$. The collection $\left\{\chi B_{1}: \chi \in \widehat{P}\right\}$ forms an open cover of $\widehat{P}$, and so by the compactness of $\widehat{P}$, there exist $\chi_{1}^{(1)}, \ldots, \chi_{k(1)}^{(1)} \in \widehat{P}$ such that $\left\{\chi_{1}^{(1)} B_{1}, \ldots, \chi_{k(1)}^{(1)} B_{1}\right\}$ forms a clopen partition of $\widehat{P}$. Furthermore, since, by Lemma 2.3, $\widehat{P} / B_{1}$ is cyclic, we label it so that $\chi_{1}^{(1)} B_{1}$ is a generator of $\widehat{P} / B_{1}$ and $\chi_{k(1)}^{(1)}$ is the identity. Likewise, we obtain a clopen subgroup $B_{2}$ of $\widehat{P}$ such that $B_{2} \subset \bar{B}_{2} \cap B_{1}$. Then, as above, there exist characters $\chi_{1}^{(2)}, \ldots, \chi_{k(2)}^{(2)} \in B_{1}$ such that $\left\{\chi_{1}^{(2)} B_{2}, \ldots, \chi_{k(2)}^{(2)} B_{2}\right\}$ forms a clopen partition of $B_{1}$. Since the sequence $\left\{\bar{B}_{n}\right\}_{n=1}^{\infty}$ is not eventually constant, we may assume, without a loss of generality, that $k(2)>k(1)$.

It follows that each of the cosets $\chi_{i}^{(1)} \chi_{j}^{(2)} B_{2}, 1 \leq i \leq k(1), 1 \leq j \leq k(2)$, is a distinct element of $\widehat{P} / B_{2}$. By Lemma 2.3, since $\widehat{P} / B_{2}$ is cyclic, we know that among these elements there exists a generator of $\widehat{P} / B_{2}$, say $\chi_{i_{0}}^{(1)} \chi_{j_{0}}^{(2)} B_{2}$. This implies that $\chi_{i_{0}}^{(1)} B_{1}$ generates $\widehat{P} / B_{1}$, and therefore there exists an integer $l, 1 \leq l<k(1)$, such that $\chi_{i_{0}}^{(1)} B_{1}=\chi_{1}^{(1)} B_{1}$. As $\chi_{1}^{(1)} B_{1}$ generates $\widehat{P} / B_{1}$, it must be that $\operatorname{gcd}(l, k(1))=1$. But then, for any $0 \leq m<k(2)$, we have $\left(\chi_{i_{0}}^{(1)} \chi_{j_{0}}^{(2)} B_{2}\right)^{l+m k(1)} \subset \chi_{1}^{(1)} B_{1}$, and therefore, the set of $k(2)$ distinct cosets

$$
\left\{\left(\chi_{i_{0}}^{(1)} \chi_{j_{0}}^{(2)} B_{2}\right)^{l+m k(1)}: 0 \leq m<k(2)\right\}
$$

must correspond to the set $\left\{\chi_{1}^{(1)} \chi_{1}^{(2)} B_{2}, \ldots, \chi_{1}^{(1)} \chi_{k(2)}^{(2)} B_{2}\right\}$ of cosets.

We leave it to the reader to show that, assuming $k(2)>k(1)$, there exists an integer $m, 0 \leq m<k(2)$, such that $l+m k(1)$ and $k(1) k(2)$ are relatively prime. Consequently, there exists an element $\chi_{1}^{(1)} \chi_{a}^{(2)} B_{2}$ of $\widehat{P} / B_{2}$ which generates $\widehat{P} / B_{2}$. We label the elements $\chi_{1}^{(2)} B_{2}, \ldots, \chi_{k(2)}^{(2)} B_{2}$ of $B_{1} / B_{2}$ such that $\chi_{1}^{(1)} \chi_{1}^{(2)} B_{2}$ generates $\widehat{P} / B_{2}$ and $\chi_{k(2)}^{(2)}$ is the identity.

These arguments can be applied inductively to obtain a sequence $\left\{B_{n}\right\}_{n=1}^{\infty}$ of nested decreasing clopen subgroups of $\widehat{P}$ with $\bigcap_{n=1}^{\infty} B_{n}=\{\mathrm{id}\}$, and such that for all $n \geq 1,\left\{\chi_{1}^{(n)} B_{n}, \ldots, \chi_{k(n)}^{(n)} B_{n}\right\}$ is a clopen partition of $B_{n-1}$ (with $B_{0}=\widehat{P}$ ), where $\chi_{k(n)}^{(n)}$ is the identity and where $\chi_{1}^{(1)} \cdots \chi_{1}^{(n)} B_{n}$ generates $\widehat{P} / B_{n}$. This follows from the fact that, for any $n \geq 1$, we can, without a loss of generality, assume that $k(n+1)>k(n) \cdots k(1)$ since the sequence $\left\{\bar{B}_{n}\right\}_{n=1}^{\infty}$ is strictly decreasing.

In analogy with the situation when $P$ is finite, we now observe that for every $n \geq 1$ and every $1 \leq i \leq k(n)$, the character $\chi_{i}^{(n)}$ can be viewed as a partial homeomorphism on $\widehat{P}$ with domain $B_{n}$. In particular, for any $\chi \in B_{n}$, this character partial homeomorphism takes $\chi$ to $\chi_{i}^{(n)} \chi$. Utilizing these character partial 
homeomorphisms, the inverse semigroup generated by the collection

$$
C=\left\{\chi_{i}^{(n)}: n \geq 1,1 \leq i \leq k(n)\right\}
$$

will be the smallest set of partial homeomorphisms on $\widehat{P}$ which contains $C$ and is closed for inverses and compositions. This inverse semigroup can be described somewhat more explicitly by defining, for all $n \geq 1$, the character partial homeomorphisms $\prod_{i=1}^{n} \chi_{s_{i}}^{(i)}: B_{n} \rightarrow \widehat{P}$ by

$$
\left[\prod_{i=1}^{n} \chi_{s_{i}}^{(i)}\right](\chi)=\chi \prod_{i=1}^{n} \chi_{s_{i}}^{(i)}
$$

where $1 \leq s_{i} \leq k(i)$, for all $1 \leq i \leq n$. It is immediate that this collection also generates the inverse semigroup. By considering the graphs of these mappings as subsets of $\widehat{P} \times \widehat{P}$, we see that the associated groupoid $\mathcal{R}$ is the union over all $n \geq 1$ of the sets

$$
\left\{\left(\chi_{s_{n}}^{(n)} \cdots \chi_{s_{1}}^{(1)} \chi, \chi_{t_{n}}^{(n)} \cdots \chi_{t_{1}}^{(1)} \chi\right): 1 \leq i \leq n, 1 \leq s_{i}, t_{i} \leq k(i), \chi \in B_{n}\right\} .
$$

This groupoid is the direct analogue of the one constructed for the finite $P$ case.

From [5, Theorem 2.7], it follows that $C^{*}(\mathcal{R})$ is AF, and we can compute the dimension group of $C^{*}(\mathcal{R})$ as the direct limit of the sequence $\mathbb{Z} \stackrel{\phi_{1,2}}{\longrightarrow} \mathbb{Z} \stackrel{\phi_{2,3}}{\longrightarrow} \cdots$ with connecting maps $\phi_{n, n+1}(x)=k(n+1) x$. We would like to address at this point the question of whether or not this method of building groupoids from characters yields a groupoid $\mathrm{C}^{*}$-algebra whose dimension group is isomorphic to $\left(G, G^{+}, u\right)$, as is the case when $P$ is finite. The answer to this question is "yes", and the next section will be devoted to proving this assertion.

\section{The Dimension group of $C^{*}(\mathcal{R})$}

We will let $H$ be the direct limit of the sequence of groups $\mathbb{Z} \stackrel{\phi_{1,2}}{\longrightarrow} \mathbb{Z} \stackrel{\phi_{2,3}}{\longrightarrow} \cdots$ mentioned above, with connecting maps $\phi_{n, n+1}(x)=k(n+1) x$. That is, we will let $\left(H, H^{+}, v\right)$ denote the dimension group of the $\mathrm{C}^{*}$-algebra $C^{*}(\mathcal{R})$. One can then readily show that

$$
H /\langle v\rangle \cong \lim _{\longrightarrow}[\mathbb{Z} /\langle k(1) \cdots k(n)\rangle] \cong \lim _{\longrightarrow} \mathbb{Z}_{k(1) \cdots k(n)},
$$

where here we have the same connecting maps $\phi_{n, n+1}(x)=k(n+1) x$ as above. Consequently, the character group of $H /\langle v\rangle$ is isomorphic to the projective limit $\stackrel{\lim }{\longleftarrow} \widehat{\mathbb{Z}_{k(1) \cdots k(n)}}$, with connecting maps $\psi_{n+1, n}: \mathbb{Z}_{k(1) \cdots k(n+1)} \rightarrow \widehat{\mathbb{Z}_{k(1) \cdots k(n)}} \widehat{\text { such that }}$ $\overleftarrow{\psi_{n+1, n}}(\chi)=\chi \circ \phi_{n, n+1}$, for all $n \geq 1$.

To prove that $\left(H, H^{+}, v\right) \cong\left(G, G^{+}, u\right)$, we begin by defining, for each $n \geq 1$, the homomorphisms $\psi_{n}: \widehat{P} \rightarrow \mathbb{Z}_{k(1) \cdots k(n)}$ by $\psi_{n}=r_{n} \circ q_{n}$. Here $q_{n}: \widehat{P} \rightarrow \widehat{P} / B_{n}$ is the quotient homomorphism and $r_{n}: \widehat{P} / B_{n} \rightarrow \mathbb{Z}_{k(1) \cdots k(n)}$ is the unique isomorphism which takes the generator $\chi_{1}^{(1)} \cdots \chi_{1}^{(n)} B_{n}$ of $\widehat{P} / B_{n}$ to the character in $\widehat{\mathbb{Z}_{k(1) \cdots k(n)}}$ which takes $x \in \mathbb{Z}_{k(1) \cdots k(n)}$ to $\exp \left[\frac{2 \pi i}{k(1) \cdots k(n)} x\right]$ in the unit circle. The following fact is a consequence of this definition for $\psi_{n}$.

Lemma 4.1. For any $n \geq 1$ and any $\chi \in \widehat{P}, \psi_{n+1, n} \circ \psi_{n+1}(\chi)=\psi_{n}(\chi)$. 
Proof. To begin, let $\chi \in \widehat{P}$, and suppose that $\chi$ is an element of the coset $\left(\chi_{1}^{(1)} \cdots \chi_{1}^{(n+1)} B_{n+1}\right)^{l+m k(1) \cdots k(n)}$, where $l$ and $m$ are integers such that $0 \leq l<$ $k(1) \cdots k(n)$ and $0 \leq m<k(n+1)$. That $\left(\chi_{1}^{(1)} \cdots \chi_{1}^{(n+1)} B_{n+1}\right)^{l+m k(1) \cdots k(n)}$ is a subset of $\left(\chi_{1}^{(1)} \cdots \chi_{1}^{(n)} B_{n}\right)^{l}$ follows immediately. Therefore, if we let $x \in \mathbb{Z}_{k(1) \cdots k(n)}$, then $\psi_{n+1, n} \circ \psi_{n+1}(\chi)(x)$ equals

$$
\begin{aligned}
\psi_{n+1, n}\left(\psi_{n+1}(\chi)\right)(x) & =\psi_{n+1}(\chi) \circ \phi_{n, n+1}(x) \\
& =r_{n+1} \circ q_{n+1}(\chi)(k(n+1) x) \\
& =\exp \left[\frac{2 \pi i(l+m k(1) \cdots k(n))}{k(1) \cdots k(n+1)} k(n+1) x\right] \\
& =\exp \left[\frac{2 \pi i l}{k(1) \cdots k(n)} x\right] .
\end{aligned}
$$

But, we also have $\psi_{n}(\chi)(x)$ equal to

$$
r_{n} \circ q_{n}(\chi)(x)=r_{n}\left(\left(\chi_{1}^{(1)} \cdots \chi_{1}^{(n)} B_{n}\right)^{l}\right)(x)=\exp \left[\frac{2 \pi i l}{k(1) \cdots k(n)} x\right] .
$$

It follows by the universal property of the projective limit that there exists a

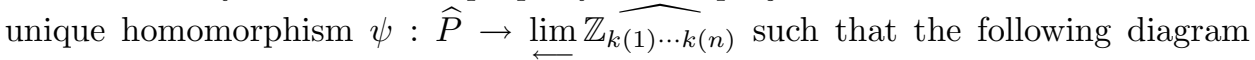
commutes:

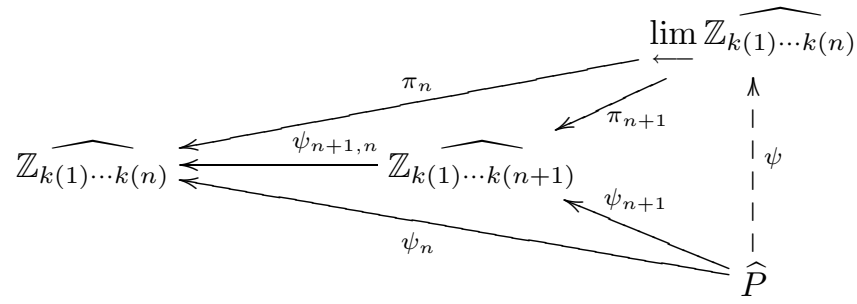

We would like to show that $\psi$ is an isomorphism.

To prove injectivity, suppose $\psi(\chi)=\mathrm{id}=\left(\mathrm{id}_{1}, \mathrm{id}_{2}, \ldots\right)$. Then, $\psi_{n}(\chi)=\pi_{n}$ 。 $\psi(\chi)=\operatorname{id}_{n}$, and so, for all $n \geq 1$ and for all $x \in \mathbb{Z}_{k(1) \cdots k(n)}$,

$$
\begin{aligned}
1 & =\psi_{n}(\chi)(x)=r_{n} \circ q_{n}(\chi)(x)=r_{n}\left(\left(\chi_{1}^{(1)} \cdots \chi_{1}^{(n)} B_{n}\right)^{l}\right)(x) \\
& =\exp \left[\frac{2 \pi i l}{k(1) \cdots k(n)} x\right],
\end{aligned}
$$

where $0 \leq l<k(1) \cdots k(n)$ and $\chi \in\left(\chi_{1}^{(1)} \cdots \chi_{1}^{(n)} B_{n}\right)^{l}$. But, this implies that $l=0$. Hence, $\chi \in B_{n}$, for all $n \geq 1$. Since $\bigcap_{n=1}^{\infty} B_{n}=\{\operatorname{id}\}$, we conclude that $\chi=$ id, and therefore, $\psi$ injects. This discussion constitutes one half of the proof of the following lemma.

Lemma 4.2. The mapping $\psi: \widehat{P} \rightarrow \widehat{\lim } \widehat{\mathbb{Z}_{k(1) \cdots k}(n)}$ is an isomorphism. 
Proof. It remains for us to prove that the homomorphism $\psi$ is in fact surjective. Let $\left(\chi_{1}, \chi_{2}, \ldots\right) \in \lim \mathbb{Z}_{k(1) \cdots k(n)}$. For each $n \geq 1$, there exists an integer $a_{n}$, $0 \leq a_{n}<k(1) \cdots k(n)$, such that for all $x \in \mathbb{Z}_{k(1) \cdots k(n)}$,

$$
\chi_{n}(x)=\exp \left[\frac{2 \pi i a_{n}}{k(1) \cdots k(n)} x\right] .
$$

Due to the fact that $\psi_{n+1, n}\left(\chi_{n+1}\right)=\chi_{n}$, for all $n \geq 1$, we have, again for all $x \in \mathbb{Z}_{k(1) \cdots k(n)}$,

$$
\exp \left[\frac{2 \pi i a_{n+1}}{k(1) \cdots k(n)} x\right]=\exp \left[\frac{2 \pi i a_{n}}{k(1) \cdots k(n)} x\right] .
$$

Therefore, we can write $a_{n+1}=a_{n}+m k(1) \cdots k(n)$, where $m$ is some integer such that $0 \leq m<k(n+1)$.

We now define the sequence $\left\{A_{n}\right\}_{n=1}^{\infty}$ of cosets in the following way. Let, for each $n \geq 1, A_{n}=\left(\chi_{1}^{(1)} \cdots \chi_{1}^{(n)} B_{n}\right)^{a_{n}}$. It follows that the sequence $\left\{A_{n}\right\}_{n=1}^{\infty}$ is nested decreasing, and so, by compactness, $\bigcap_{n=1}^{\infty} A_{n}$ is nonempty. Assume $\chi, \bar{\chi} \in$ $\bigcap_{n=1}^{\infty} A_{n}$. Thus, for all $n \geq 1, \chi$ and $\bar{\chi}$ are elements of the same coset in $\widehat{P} / B_{n}$. Hence, for all $n \geq 1, \bar{\chi} \chi^{-1} \in B_{n}$, and therefore, $\bar{\chi} \chi^{-1}=$ id. Thus, $\bigcap_{n=1}^{\infty} A_{n}$ is in fact a singleton, and we let $\chi$ denote the lone element in this intersection.

Now, $\pi_{n} \circ \psi(\chi)=\psi_{n}(\chi)=r_{n} \circ q_{n}(\chi)$, and so, for all $x \in \mathbb{Z}_{k(1) \cdots k(n)}$,

$$
\pi_{n} \circ \psi(\chi)(x)=\exp \left[\frac{2 \pi i a_{n}}{k(1) \cdots k(n)} x\right]=\chi_{n}(x) .
$$

Thus, $\psi(\chi)=\left(\chi_{1}, \chi_{2}, \ldots\right)$, and the proof is complete.

It is straightforward to establish that $\psi$ is also a homeomorphism.

Now, because, as topological groups, we have $\widehat{P} \cong \lim \mathbb{Z}_{k(1) \cdots k(n)}$, we can apply the Pontryagin Duality Theorem to obtain

$$
G /\langle u\rangle=P \cong \lim _{\longrightarrow} \mathbb{Z}_{k(1) \cdots k(n)} \cong H /\langle v\rangle .
$$

By Lemma 2.2 we know that $\left(G, G^{+}, u\right)$ is linearly ordered, as is $\left(H, H^{+}, v\right)$. Therefore, it will follow that $\left(G, G^{+}, u\right) \cong\left(H, H^{+}, v\right)$. One can then immediately state the following corollary.

Corollary 4.3. Let $\mathfrak{A}$ be an AF algebra and let $\left(G, G^{+}, u\right)$ be the corresponding dimension group. Then $G /\langle u\rangle$ is a torsion group if and only if $\mathfrak{A}$ is a UHF algebra.

Further work is needed to extend these results to more general dimension groups. In general the group $P=G /\langle u\rangle$ will not be a torsion group. Therefore, $\widehat{P}$ does not provide the correct unit space as it does above. It may be that the subgroup $\langle u\rangle$ needs to be expanded in some way to include more elements of $G$. A question of more limited scope would involve how to deal with an arbitrary linearly ordered dimension group, as there are such dimension groups for which the results of the present paper do not apply, i.e. the Fibonacci algebra (see [1]).

\section{ACKNOWLEDGEMENT}

The author thanks an anonymous reviewer for a number of helpful suggestions. 


\section{REFERENCES}

1. Kenneth R. Davidson, $C^{*}$-algebras by example, Fields Institute Monographs, vol. 6, American Mathematical Society, Providence, Rhode Island, 1996. MR.1402012 (97i:46095)

2. G. A. Elliott, On the classification of inductive limits of sequences of semisimple finitedimensional algebras, J. Algebra 38 (1976), no. 1, 29-44. MR0397420 (53:1279)

3. E. Hewitt and K. A. Ross, Abstract harmonic analysis I, 2nd ed., Grundlehren der math. Wiss., vol. 115, Springer-Verlag, Berlin, Heidelberg, New York, 1979. MR0551496 (81k:43001)

4. Alan L. T. Paterson, Groupoids, inverse semigroups, and their operator algebras, Progress in Mathematics, vol. 170, Birkhäuser, Boston, 1999. MR.1724106 (2001a:22003)

5. Justin R. Peters and Ryan J. Zerr, Partial dynamical systems and AF $C^{*}$-algebras, Houston J. Math. 31 (2005), no. 2, 463-494. MR.2132848

6. Stephen C. Power, Limit algebras: An introduction to subalgebras of $C^{*}$-algebras, Pitman Research Notes in Mathematics, vol. 278, Longman Scientific and Technical, Harlow, Essex, England, 1992. MR 1204657 (94g:46001)

7. Jean N. Renault, A groupoid approach to $C^{*}$-algebras, Lecture Notes in Mathematics, vol. 793, Springer-Verlag, Berlin, Heidelberg, New York, 1980. MR0584266 (82h:46075)

Department of Mathematics, University of North Dakota, Grand Forks, North DАКОтА 58202

E-mail address: ryan.zerr@und.nodak.edu 